\title{
SMART SHOPPING SYSTEM
}

\author{
Pratyaksha Ambast, Nandini Yadav, Rahul Kumar Singh \\ Department of Information Technology , \\ Thakur College of Engineering and Technology (TCET) \\ Kandivali East, Mumbai - 400101
}

pratyakshaambast2000@gmail.com, nandini976804@gmail.com, singhrahulkumar564@ gmail.com

\begin{abstract}
It has been seen that Supermarkets witness long lines during the pinnacle of the shopping period. These long lines have brought about the loss of time both for the customers also, the shopping center chaperons. Thus, the objective of this paper is to plan and build up an Intelligent Smart Shopping Cart with the sole goals of limiting shopping time and boosting shopping involvement with a general store. This paper presents an Intelligent Smart Shopping Cart System where the aggregate sum the customer needs to spend is ascribed into the smaller than usual framework implanted in the shopping trolley. Each item picked has an RFID label which has a doled-out cost. At the point when the items dropped in the brilliant shopping basket come to the greatest information sum or surpass the most extreme information sum, the red visual pointer begins to flicker and the ringer signals to advise the customers of surpassing as far as possible while shopping. The green visual pointer additionally flickers when a customer shops beneath the attributed sum. In the event that any item is eliminated from the shopping trolley, the sum is deducted from the created small framework implanted in the shrewd shopping basket and the ringer likewise blares to advise the Shopper of the derivation. The segments utilized for this exploration are RFID Tag, RFID Reader, Liquid Crystal Display (LCD), Push catches/Switch, Reset button, Visual Indicator, Wi-Fi module also, a PIC microcontroller.
\end{abstract}

Keywords: RFID Tags, Reader, LCD, smart-cart, navigation, book-a-slot, scan.

\section{Introduction}

Technology has improved rapidly and looking at the after COVID situation, people are mostly reliable on tools and application through software. Mobile application has been increasingly dominating from healthcare to financial banking to shopping online. Everything is accessible through one click. Due to the pandemic, people will avoid going to crowded place and would look a more convenient way for shopping. Grocery and essential shopping are the needs of day-to-day chores and in order to adapt time saving measure, technology has proven to be a boon. Even though online shopping has been in demand, local customers still prefer offline shopping to check the product quality and buy stuff conveniently. But this method is tedious as people have to wait in long queues for their product to be scanned by cashier also the crowd surrounded at the time of shopping. This motivated us to invent a smart shopping application which will meet all its requirement and will make the shopping time saving and feasible.

\section{Motivation}

The society has drastically changed because of the advancement in the field of technology. The adoption of various technologies has simplified our day-to-day chores like accessing education, medicine, transportation, and even shopping. By using smart applications, it has saved us a lot of time and effort. When we took a survey, it was found that the maximum people who go for outdoor shopping are mainly between 23 to 50 years of age and the main problem that they face is a crowded place and time that has been spent waiting in long queues. But when it comes to choosing between online shopping and offline shopping, there is a 50-50 choice as people are mainly concerned with product quality and return policy in online shopping. This concern of the people led us to an idea where they can get the benefits of online shopping in outdoor shopping and hence avoid issues like crowded areas, waiting in long queues, or security breaches. By using our application, the user will have various options to explore like to book a slot, to create a list of the items they want to buy, easily navigate a particular product in the crowded supermarket, get notification of recent discounts and sales, and easily pay for the products they have bought without even standing in the queue. This will motivate people to opt for a supermarket experience that has been declining in the past few years.

\section{Related Work}

Automatic Bill Generation of Products using digital

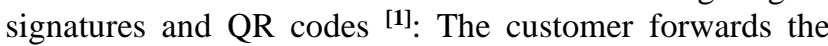
selected product list to the server and the response received enables the consumer to decide based on the authenticity. In a real time capturing system using QR code in Android smart phone, QR code verifies products by capturing it 
through the smart phone, then decodes and sends it to the server for authentication.

Smart Shopping using Smart Trolly [2]: Whenever the customer puts a product into trolley, it will get scanned by RFID reader/Scanner and product price and cost will be displayed on LCD display. It is less time consuming as compared to regular billing technique. It is more reliable and provides ease for shopping.

Smart Shopping Cart for Automatic Billing in Supermarket [3]: This RFID system can be used in shopping malls and can be used for security applications by keeping data confidential. The chip and antenna are sandwiched between a printed label and its adhesive backing is inserted into a more durable structure.

Smart Shopping - An android based shopping application [4]: Customer scans the unique code at the entry of retail shop, after which the items are scanned and details from the database are accessed once sent. The barcode which is read will be decoded and then converted into any specific number that will be unique for every product after customer has scanned the barcode; a web service will retrieve data from the database

Design and Implementation of an Android Application for Smart Shopping ${ }^{[5]}$ : The user is asked to connect the device to the shopping cart. On pressing the 'connect' button the application checks for nearby devices whose Bluetooth is enabled. The devices get paired to it and the connection is established. Window is aided with RFID the buttons in 'manual' control page are programmed to send data streams to the shopping cart via Bluetooth. Each button sends a different set of data. Based on the received data, the microcontroller can process the signals and facilitate movement of the cart.

Improvised Smart Shopping Based on Android Application [6]: This Application is designed with basis of two mode online (inside the shop) and offline (outside the shop) in each scenario the implementation is described with facilities of payment, offers, invoice generation and history of purchase. It makes use of barcode scanning. The application is designed to work in the online mode if and only if the mobile is connected to the particular $\mathrm{Wi}-\mathrm{Fi}$ and the battery life is greater than $25 \%$. If the customer in unable to scan the barcode due to various reasons the code can be manually entered.

\section{System Architecture}

Our system is designed to aim at the shopping malls. The user has to authorize himself/herself by registering in the app and logging in at the time of shopping. While user has reached his/her destination for the shopping, the app will prompt for a to-do-list option, for the user to quickly make a list of items for purchase. If the user doesn't want, they can skip the option for later use. Then there is a navigation option which will allow users to navigate to a particular product, while he/she is purchasing our app will recommend similar product in vicinity or through to-do-list and will remind user to buy the product. User can also get the product details by clicking on that particular product. The user then has to scan the barcode by himself/herself to ensure the product they selected. If the scanner doesn't work, the user can manually enter the barcode number. In the setting options, user have to include their payment details for automatic payment after scanning the barcode. If the transaction fails due to any reason, then the user will be informed and it will be taken care. If user tries to go out without paying for the product via integrated payment method, the retailers can check upon it as our application will make a beep sound which will be an indication of failure of payment, in this way security has been taken care. Before going to shopping mall, user can check through app if there is an availability for shopping to avoid crowd. Using session for each user, app will generate a token for that particular user who wants to shop and through which the shopping mall vendors can allot a specific time for users to enter. In this way social distancing can be taken care and people don't have to wait in long queues for their turn. There will also be a feedback and review system in app to know user's satisfaction and infelicities which can be improve in further development.

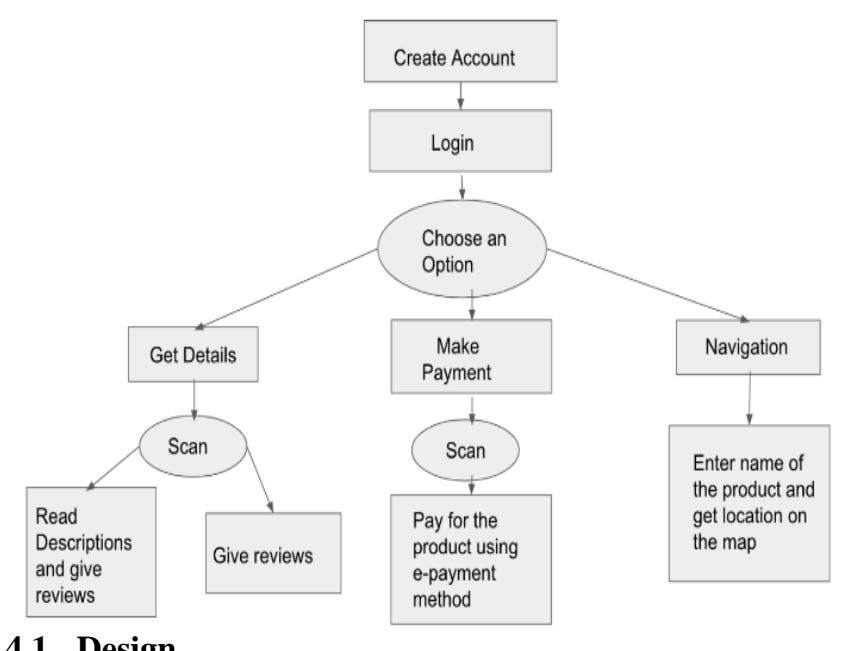

\subsection{Design}

The design and layout of our application is discussed in this section. The app has 5 major features like book your slot, bill, scan, navigate, and create a shopping list. In book your slot feature, the user can book a time as per their convenience and do shopping without waiting in the long queue. In the bill feature, the user has to scan a product and subsequently pay for it using the integrated e-payment system or if the scan feature is not working, the user can then enter the barcode manually and proceed for payment. 
Instead of standing in long queues, one can save time by using this app and has the option of online payment so no need to worry about the change of money. With the scan option, one can scan the product and gather all the necessary information like ingredients present in a food product or if the product is tech-related then check for made in which country and warranty period. In the navigate option, the user can easily spot a particular product in the crowded supermarket. This will avoid wasting time in searching for the product and the user can easily find the necessary item using this navigation tool on our application. In the create a shopping list option, the user can jot down their products that they want to buy so the app will shuffle the products according to the arrangements of items placed inside the supermarket. This will ease the shopping for the user and avoid missing any product off the list. An RFID reader controlled by Arduino is used in this automated payment system. The RFID module detects the product when it is put in the trolley and displays its price on the LCD screen. The module detects all products as the shopper adds them, thus raising the price accordingly. Any product that client decides not to add to the trolley after it has been added may be removed and the price added will be deducted automatically.

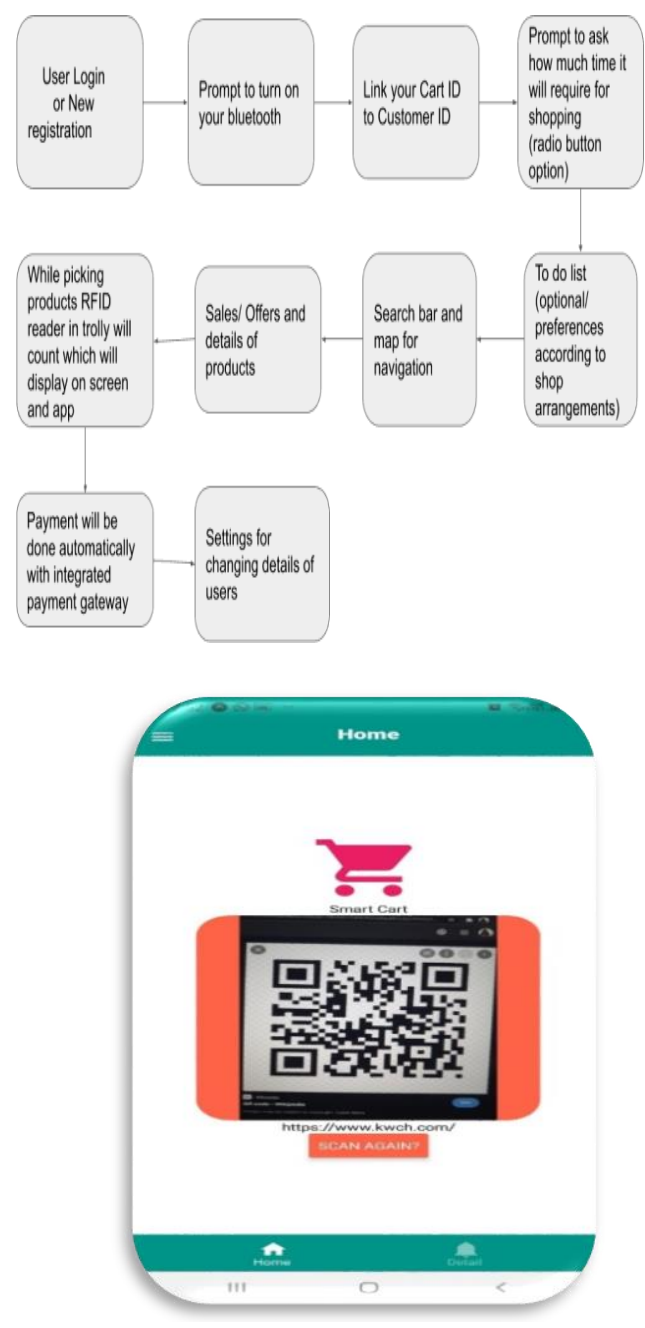

\section{Implementation}

The flow of the implementations starts with User Login if the person is already registered or else, they have to register to use the app. Once the user is logged in, they are prompted to turn on their Bluetooth this feature is used to determine the proximity of the products as well as other users within the range. The registered users should link their Customer ID to the Cart ID which they are using. In order to keep track of time taken by the customers they are prompted to choose the time limit in the app, after that they can proceed their way to do the purchase. There will be an optional feature as 'To do list' which can be set by the customers according to the arrangement of the products in the shop. They can be able to list their products which they are going to buy in the list and can proceed accordingly. Users can also search the products using search bar and see them on the map for navigation. It also shows them the sales and offers in that particular shop.

The RFID chip which is embedded in the trolly will read the product details and count them when a customer put them inside the trolly after that all the details will be displayed on the app. They can also remove a product from the trolly and the same will be reflected in the app. After the completion of all the purchase, payment will be done through integrated payment gateways and a bill will be generated for the same.

\section{Conclusion}

Our project "Smart Shopping System", as it suggests and tries to offer a smart application to mitigate users' difficulties while shopping. This system targets on reducing the shopping time of customers by automated payment integration through which customers don't have to wait in queue for long hours. We conclude that our system is time-saving with faster checkout process. Also, this reduces the labor requirement at the counter.

\section{Future Works}

The word smart means to be well equipped and be well versed in situations. Hence the future works are aimed at incorporating the following features into the application. A customized user interface will be provided to the app with elevated features. To make the app more productive, hosting it on cloud platforms will be investigated. The app will be designed to suggest personalized offers and discounts to users based on customer shopping patterns as soon as the customer enters a particular supermarket. Security can be improved by using a camera module and sensors, which scans the weight of the cart and products and automatically checks if any product is left to scan and pay for it. 


\section{Acknowledgement}

We would like to express our sincere gratitude to all the students involved in writing this research paper. Our sincere thanks to the immense support extended by the Information Technology Department and our teacher Miss. Mary Margaret Valentine for guiding us through the project. Besides, we are grateful to our parents for always supporting and encouraging us with their blessings.

\section{References}

[1] Thakur Prerana, Shikha Ranjan, Prachi Kaushik, 2017 - Smart Shopping Cart for Automatic Billing in Supermarket.

[2] S. Kalarani Professor Department of IT, 2017 - An Automatic Bill Generation of Products using digital signatures and QRcodes

[3] Ghatol Sonali Digambar, Mrs.V.S.Jahagirdar ,Pratiksha Dattatraya Khamitkar, 2018 - Smart Shopping using SmartTrolly

[4] Adarsh Borkar Madhura Ansingkar Monali Khobragade Pooja Nashikkar Arti Raut March 2015 Smart Shopping - An android based shopping application

[5] Alfred.P.F 7-May 2016 - Improvised Smart Shopping Based on Android Application

[6] Rajesh Kannan Megalingam, Souraj Vishnu, Swathi Sekhar, Vishnu Sasikumar, Sreekumar S and Thejus R Nair April 4-6 2019 - Design and Implementation of an Android Application for Smart Shopping 